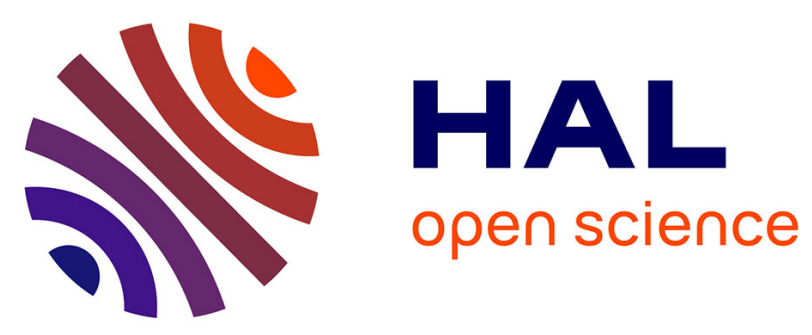

\title{
Etude d'un revêtement d'acier inoxydable Z 2 CND 18-12 réalisé sur un acier doux, sous irradiation laser avec injection de poudre coaxiale au faisceau
}

F. Fouquet, P. Sallamand, P. Dierickx, D. Bonne, J. Millet

\section{- To cite this version:}

F. Fouquet, P. Sallamand, P. Dierickx, D. Bonne, J. Millet. Etude d'un revêtement d'acier inoxydable Z 2 CND 18-12 réalisé sur un acier doux, sous irradiation laser avec injection de poudre coaxiale au faisceau. Journal de Physique IV Proceedings, 1994, 04 (C4), pp.C4-85-C4-88. 10.1051/jp4:1994417 . jpa-00252627

\section{HAL Id: jpa-00252627 https://hal.science/jpa-00252627}

Submitted on 1 Jan 1994

HAL is a multi-disciplinary open access archive for the deposit and dissemination of scientific research documents, whether they are published or not. The documents may come from teaching and research institutions in France or abroad, or from public or private research centers.
L'archive ouverte pluridisciplinaire $\mathbf{H A L}$, est destinée au dépôt et à la diffusion de documents scientifiques de niveau recherche, publiés ou non, émanant des établissements d'enseignement et de recherche français ou étrangers, des laboratoires publics ou privés. 


\title{
Etude d'un revêtement d'acier inoxydable Z 2 CND 18-12 réalisé sur un acier doux, sous irradiation laser avec injection de poudre coaxiale au faisceau
}

\author{
F. FOUQUET, P. SALLAMAND, P. DIERICKX, D. BONNE and J.P. MILLET*
}

GEMPPM-CALFETMAT, INSA, Bât. 502, 69621 Villeurbanne cedex, France

${ }^{*}$ Physicochimie Industrielle, INSA, Bât. 401, 69621 Villeurbanne cedex, France

\begin{abstract}
RESUME
Le présent travail traite du dépôt d'acier inoxydable austénitique de type AISI 316L sur acier doux par projection de poudre sous faisceau laser et de la caractérisation du revêtement élaboré. Les revêtements obtenus par cette technique sont de bonne qualité, exempts de fissures, avec peu de porosités et parfaitement adhérents au substrat. Leur microstructure très fíne est dendritique ou cellulaire. La structure est apparue majoritairement austénitique, mais avec présence non négligeable de ferrite $\delta$ localisée aux joints de grains d'austénite. La résistance à la corrosion, tant généralisée que par piqûre en solutions salines aqueuses, s'est avérée excellente.
\end{abstract}

\section{INTRODUCTION}

L'utilisation de l'outil laser pour la réalisation de revêtements résistant à la corrosion a fait l'objet de nombreuses études $[1,2,3]$. Dans de précédents travaux, nous avons étudié des revêtements d'aciers inoxydables austénitiques sur des substrats d'acier doux, réalisés soit par fusion de prédépôts nickel-chrome [4], soit par projection de poudre [5]. Si cette dernière technique a permis d'obtenir sans difficultés des revêtements d'acier inoxydable de type AISI 304 de bonne qualité, les revêtements de type AISI 316 obtenus étaient systématiquement fissurés [5]. Pour expliquer le phénomène de fissuration observé dans ce dernier cas, l'hypothèse d'une fissuration à chaud de l'acier, résultant d'une solidification primaire en phase $\gamma$, a été retenue. La teneur élevée en carbone de la poudre utilisée $(\approx 0,1 \%)$ nous a conduit à reprendre cette étude avec une poudre d'acier de même type, mais à très faible teneur en carbone $(0,02 \%)$, afin de vérifier l'hypothèse précédente et d'éliminer la fissuration. C'est l'objet du présent travail, pour lequel, outre la composition de la poudre, la technique de projection a également été modifiée.

\section{METHODE EXPERIMENTALE}

La composition de la poudre d'acier inoxydable utilisée, de nuance $316 \mathrm{~L}$, est donnée dans le tableau 1; sa granulométrie est comprise entre 50 et $100 \mu \mathrm{m}$. Le substrat est un acier non allié à bas carbone. Les échantillons sont de forme parallélipipédique $\left(80 \times 40 \times 10 \mathrm{~mm}^{3}\right)$. Le laser utilisé est un laser $\mathrm{CO}_{2}$ continu de puissance maximum $3,5 \mathrm{~kW}$.

La buse, permettant l'injection de la poudre coaxialement au faisceau laser, est représentée sur la figure 1. Son principe est fondé sur l'association de deux vortex antagonistes, l'un - le vortex supérieur - jouant le rôle de protection des optiques et de frein du second, l'autre - le vortex inférieur - servant de vecteur au jet de poudre fluidisé [6]. Cette buse est pour l'essentiel composée de deux cônes directeurs permettant la création de vortex tournant en sens opposés. Le gaz injecté est de l'argon. Les paramètres géométriques ainsi que les paramètres d'injection du gaz ont été optimisés de façon à obtenir un bon confinement du jet de poudre en sortie de buse. Ce dispositif d'injection axiosymètrique présente l'avantage, par rapport aux injecteurs latéraux classiques, de pouvoir réaliser de façon simple des dépôts multidirectionnels et d'obtenir des rendements de projection de poudre plus élevés.

Les revêtements sont réalisés en déplaçant la pièce sous le faisceau selon un processus de "traitement multipasses" (figure 1). Le taux de recouvrement entre passes est d'environ $60 \%$. 
Tableau 1 : Composition moyenne de la poudre d'acier $316 \mathrm{~L}$ et des revêtements (\% poids)

\begin{tabular}{|l|c|c|c|c|c|c|c|c|}
\hline & $\mathrm{Fe}$ & $\mathrm{Cr}$ & $\mathrm{Ni}$ & $\mathrm{Mo}$ & $\mathrm{Mn}$ & $\mathrm{Si}$ & $\mathrm{C}$ & autres \\
\hline poudre 316L & 64,8 & 19,3 & 11,5 & 2,5 & 1,2 & 0,5 & 0.02 & $\approx 0,2$ \\
\hline revêtement & 65,0 & 19,2 & 11,1 & 2,3 & 1,3 & 0,6 & - & $\approx 0,5$ \\
\hline
\end{tabular}

Les paramètres de traitement ont été optimisés en faisant varier la vitesse de balayage de la pièce $\mathrm{Vb}$ de 400 à $800 \mathrm{~mm} \cdot \mathrm{min}^{-1}$ et la puissance laser $\mathrm{P}$ de 1,2 à $1,8 \mathrm{~kW}$, les autres paramètres (diamètre du faisceau d'environ $2 \mathrm{~mm}$, diamètre de sortie de la buse d'injection de $4 \mathrm{~mm}$, débit

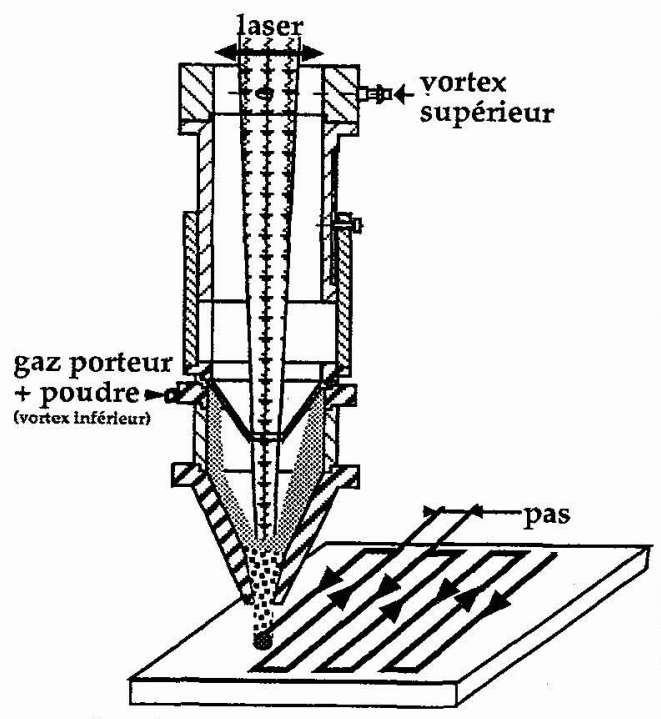

Figure 1 : Représentation schématique de la buse d'injection de poudre de 5 g.min $\mathrm{min}^{-1}$ et distance interpasses de $0,9 \mathrm{~mm}$ ) étant fixes. Quelles que soient les valeurs de $\mathrm{Vb}$ et $\mathrm{P}$ utilisées, les revêtements obtenus n'ont présenté aucune fissuration. Un revêtement d'épaisseur régulière $(600$ à $700 \mu \mathrm{m})$ et présentant très peu de porosités a été réalisé avec $P=1,8 \mathrm{~kW}$ et $\mathrm{v}=600 \mathrm{~mm} / \mathrm{min}$.

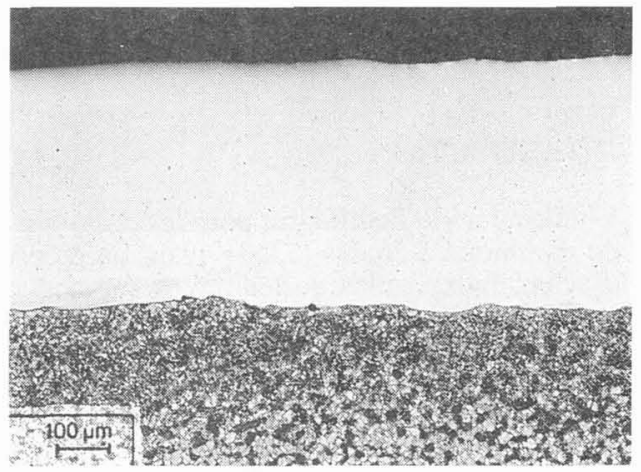

Figure 2 : Micrographie optique d'une coupe transversale (attaque nital 3\%)

\section{CARACTERISATION MICROSTRUCTURALE}

Une coupe transversale d'un échantillon (fig. 2) fait apparaître un revêtement d'épaisseur assez uniforme ne contenant que quelques porosités et exempt de fissures. L'étude micrographique montre également une fusion du substrat sur une épaisseur très faible (quelques $\mu \mathrm{m}$ ), mais néanmoins suffisante pour assurer une bonne adhérence avec le substrat. Cette fusion extrêmement limitée est confirmée par la microanalyse EDS qui fournit pour le dépôt une composition chimique moyenne quasi-identique à celle de la poudre projetée (tableau 1). Les profils de concentration des principaux éléments $(\mathrm{Fe}, \mathrm{Cr}, \mathrm{Ni})$ perpendiculairement à la surface du revêtement (fig. 3) révèlent par ailleurs une bonne homogénéité de composition chimique dans toute l'épaisseur de la couche déposée. Ces profils montrent le changement brutal de composition à l'interface substrat-revêtement, confirmant clairement la faible dilution.

Les études en diffraction $X$ font apparaître (fig. 4) une structure austéno-ferritique du revêtement. Les observations micrographiques (fig. 5 et 6 ) confirment ce résultat et permettent de voir que la ferrite $\delta$ se présente sous forme d'ilôts plutôt localisés aux joints triples des grains d'austénite. La microstructure très fine se révèle être dendritique ou cellulaire et changeante à 
l'intérieur du revêtement. Les interfaces entre cordons apparaissent comme des zones perturbées avec des directions de croissance dendritique variables. Au sein d'un cordon les dendrites ou cellules croissent à partir du substrat ou de l'interface avec le cordon précédent, en direction de la surface dans le sens du gradient thermique.

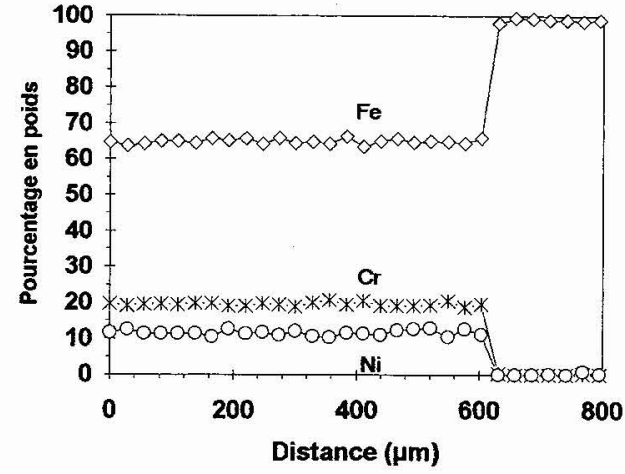

Figure 3 : Profils de concentration en $\mathrm{Fe}, \mathrm{Cr}$, $\mathrm{Ni}$, perpendiculairement à la surface du revêtement (microanalyse EDS)

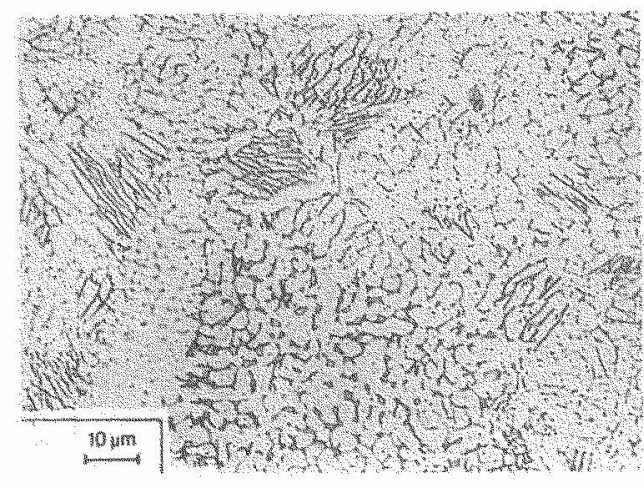

Figure 5 : Micrographie optique de la surface du revêtement (attaque réactif de Catella)

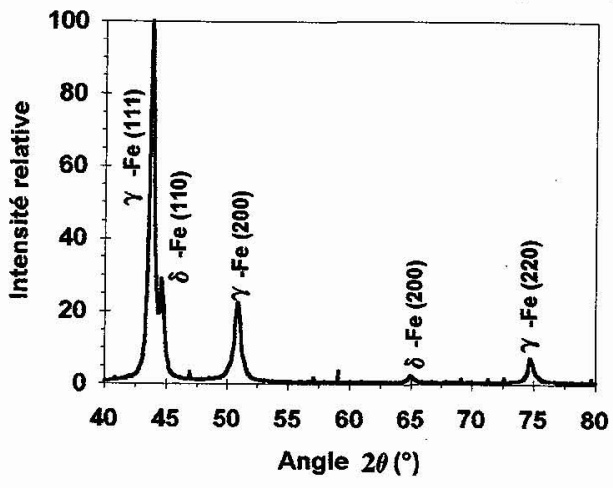

Figure 4 : Diagramme de diffraction $\mathrm{X}$

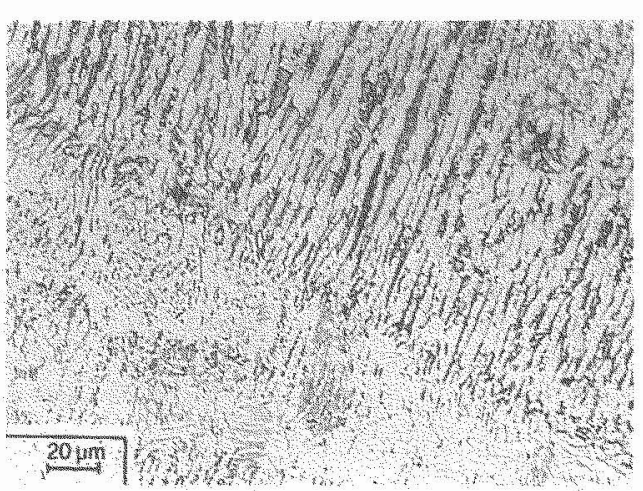

figure 6: Micrographie optique sur une section transversale (attaque réactif de Catella)

La ferrite $\delta$ n'est pas uniformément répartie de part et d'autre des interfaces entre cordons. Cette non uniformité peut s'expliquer par l'augmentation de la vitesse de solidification à l'intérieur d'un cordon du début à la fin de la solidification conduisant à une diminution progressive de la quantité de ferrite $\delta$ formée. On peut également l'interpréter en considérant que, dans chaque cordon, dans la zone affectée thermiquement lors de la réalisation du cordon suivant, une transformation $\alpha \rightarrow \gamma$ se produit et que la phase $\gamma$ est hypertrempée lors du refroidissement.

L'absence de fissures dans les revêtements réalisés avec la poudre d'acier inoxydable $316 \mathrm{~L}$ associée à la présence de ferrite $\delta$ dans ces revêtements semble confirmer l'hypothèse faite précédemment [5] de l'existence d'une fissuration à chaud si la structure de solidification est totalement austénitique. Une solidification primaire non négligeable de ferrite $\delta$ apparaît donc comme nécessaire pour éviter la fissuration des revêtements d'acier inoxydable austénitique de type 316 et qu'en conséquence les nuances à très bas carbone sont à retenir. 


\section{RESISTANCE A LA CORROSION}

Le comportement à la corrosion des matériaux élaborés a été testé en milieu $\mathrm{NaCl}$ aqueux $30 \mathrm{~g} / \mathrm{l}$, naturellement aéré ou désaéré par barbotage d'azote pendant $30 \mathrm{~min}$. avant l'essai. Des enregistrements potentiel-temps, suivis de tracés voltampéromètriques, avec vitesse de balayage de $1 \mathrm{mV} / \mathrm{s}$, ont conduit à déterminer le potentiel de corrosion Ecor et la densité de courant de corrosion Icor ; celle-ci permet le calcul de la vitesse de corrosion en mm/an. Des essais à vitesse de balayage très lente ont permis de déterminer le potentiel d'initiation de piqûre de ces revêtements passivables. Les résultas de ces essais ont été regroupés dans le tableau 2, dans lequel figurent également les données relatives à un acier AISI 316L massif, pour comparaison.

Tableau 2 Résultats des mesures électrochimiques pour 2 échantillons et 316L massif (conditions d'élaboration : (1) $P \approx 1700 \mathrm{~W}, \mathrm{Vb}=60 \mathrm{~cm} / \mathrm{min}-(2) \mathrm{P} \approx 1800 \mathrm{~W}, \mathrm{Vb}=60 \mathrm{~cm} / \mathrm{min}$ )

\begin{tabular}{|c|c|c|c|c|c|c|c|}
\hline & \multicolumn{3}{|c|}{$\mathrm{NaCl} 30$ g.: $\mathrm{L}^{-1}$ désaéré } & \multicolumn{4}{|c|}{$\mathrm{NaCl} 30 \mathrm{~g} . \mathrm{I}^{-1}$ aéré } \\
\hline & $\begin{array}{c}\mathrm{E}_{\text {cor }} \\
\mathrm{mV} / \mathrm{ECS}\end{array}$ & $\begin{array}{c:c}\mathrm{E}_{\text {piqûre }} & \Delta \mathrm{E}^{\star} \\
\mathrm{mV} / \mathrm{ECS} & \mathrm{mV}\end{array}$ & $\begin{array}{c}I_{\text {cor }}^{\#} \\
\mu \mathrm{A} / \mathrm{cm}^{2}\end{array}$ & $\begin{array}{c}\mathrm{E}_{\mathrm{cor}} \\
\mathrm{mV} / \mathrm{ECS}\end{array}$ & $\begin{array}{c}\mathrm{E}_{\text {piqûre }} \\
\text { imV/ECS i }\end{array}$ & $\underset{\mathrm{mV}}{\Delta \mathrm{E}^{*}}$ & $\begin{array}{r}I_{\text {cor }}^{\#} \\
\mu \mathrm{A} / \mathrm{cm}^{2}\end{array}$ \\
\hline $\begin{array}{l}\text { AIST3I6L } \\
\text { massif } \\
\text { echantillon } 1 \\
\text { revêtement } \\
\text { type AISI 316L } \\
\text { échantillon } 2 \\
\text { revêtement } \\
\text { type AISI 316L }\end{array}$ & $\begin{array}{l}-576 \\
-470\end{array}$ & 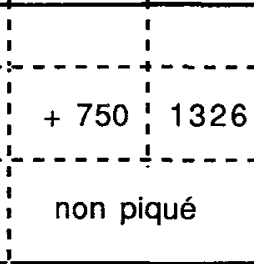 & \begin{tabular}{cc} 
\\
$:$ & \\
\hdashline & 0,8 \\
\hdashline & 0,8
\end{tabular} & $\begin{array}{c}-164 \\
---- \\
-177 \\
----- \\
-160\end{array}$ & $\begin{array}{r}\text { non } \\
1+703 \\
\text { non } p\end{array}$ & 880 & 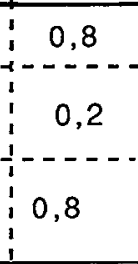 \\
\hline
\end{tabular}

\section{CONCLUSION}

La technique d'élaboration de dépôts par projection d'un jet de poudre coaxialement au faisceau laser a permis d'obtenir des revêtements de bonne qualité, exempts de fissures, contenant peu de porosités et présentant une bonne adhérence avec le substrat. La structure est majoritairement austénitique, mais avec une quantité significative de ferrite $\delta$. La microstructure très fine est de type dendritique ou cellulaire, la ferrite $\delta$ étant localisée dans les espaces intercellulaires. La solidification primaire de ferrite $\delta$ apparaît bien comme un élément déterminant pour éviter la fissuration à chaud des revêtements d'acier inoxydable austénitique. L'étude du comportement à la corrosion en milieu salin a montré que les densités de courant de corrosion restent très faibles, dans les milieux étudiés, et que le potentiel d'initiation de piqûres est élevé. Ceci traduit l'excellente résistance à la corrosion uniforme et à la piqûre des matériaux élaborés.

\section{BIBLIOGRAPHIE}

[1] Mc CAFFERTY E, HUBLER G.V., NATISHAN P.M, MOORE P.G, KANT R.A. and SARTWELL B.D., Mat. Sci. and Eng. 86 (1987) 1.

[2] CHIBA S., SATO T., KAWASHIMA A, ASAMI K. and HASHIMOTO K., Corrosion Science 26 (1986) 311.

[3] WEERASINGHE V.M, STEEN W.M, WEST D.R.F, Surface Engineering 3 (1987) 147

[4] FOUQUET F., RENAUD L., MAZILLE H., CROLET J.L., Mem. Et. Sc. Rev. Met.2 (1992), 103.

[5] FOUQUET F., SALLAMAND P., MILLET J.P., FRENK A., WAGNIERE J.D., 3ème Conférence Internationale LASER M2P - Lyon décembre 1993.

[6] MARCHIONE T., FRENEAU D., LAGRANGE L., VANNES A.B., KINKELIN K., BERGER V., WEISBROD J., DEZERT D., Proc. 5ème Colloque International sur le soudage et la fusion par faisceau d'électrons et laser, juin 1993 - La Baule, p 147. 